\title{
IDENTIFICAÇÃO DA MICROBIOTA FÚNGICA DE AMBIENTES CONSIDERADOS ASSÉPTICOS
}

\author{
Maria Elisabete Sbrogio de Almeida* \\ Maria Helena Martini* \\ Edward Porto* \\ Ana Maria Martins de Camargo* \\ Edda de Rizzo* \\ Carlos da Silva Lacaz**
}

\begin{abstract}
ALMEIDA, M.E.S. de et al. Identificação da microbiota fúngica de ambientes considerados assépticos. Rev. Saúde públ., S. Paulo, 22: 201 - 6, 1988.

RESUMO: Objetivou-se isolar e identificar a microbiota fúngica em ambientes considerados assépticos, através de exposiçóes com meios de cultivo adequados, em três épocas distintas do ano, antes e imediatamente após as manobras técnicas realizadas em três áreas de trabalho: ambiente aberto, ambiente fechado sem filtração de ar e ambiente fechado com filtração de ar, utilizadas em produçăo de imunobiológicos. Os meios ágar-Sabouraud e ágar-soja, enriquecidos com $0,2 \%$ de extrato de levedura e sem cloranfenicol, foram estudados quanto à sua eficácia no isolamento de bolores e leveduras, considerando-se o número de colônias desenvolvidas e a freqüência dos diversos fungos isolados. Isolaram-se 67 espécimens, sendo 64 fungos filamentosos (bolores) e três leveduras. Dos bolores, 54 pertenciam a 22 gêneros da divisão Deuteromycota, famflias Moniliacere e Dematiaceac, cinco amostras filamentosas foram incluídas na ordem Agonomycetales (Mycelia Sterilia), e uma amostra foi classificada na divisão Deuteromycota, ordem Sphaeropsidales, classe Coelomycetes. Da divisão Zygomycota, ordem Mucorales, familia Mucoraceae, um único mucoráceo foi identificado até gênero. As três leveduras pertenciam também à divisão Deuteromycota (Fungi Imperfecti), familia Cryptococcaceae, e foram ideritificadas como sendo duas Rhodotorula rubra e uma Torulopsis candida. Comprovou-se que o número de colônias isoladas aumentou após a realização das monobras técnicas e que a filtração de ar através de filtros tipo HEPA, reduzindo o número de colônias isoladas nos ambientes fechados, aumenta a segurança do trabalho; comumente é recomendada para áreas de atividade técnica cujos resultados satisfatórios estão diretamente relacionados com uma baixa incidência de contaminantes.
\end{abstract}

UNITERMOS: Fungos, isolamento. Leveduras, isolamento. Ambiente construído. Assepsia. Microbiologia ambiental.

\section{INTRODUÇÃO}

Em laboratorios de pesquisa ou de produção de imunobiológicos, todas as operaçőes técnicas devem ser realizadas em ambientes controlados quanto à sua esterilidade. A não-observância deste requisito pode dar origem a contaminaçðes por bactérias e fungos, que determinam 0 descarte do produto, impedindo o cumprimento dos compromissos de produçăo assumidos e alterando a relaçăo de custo prevista. Os testes de rotina para a comprovação da esterilidade de produtos imunobiológicos e/ou farmacêuticos são feitos com o meio de cultura caldo de soja ${ }^{10,11}$, recomendado pela Organização Mundial da Saúde ${ }^{12}$ pelo Public Health Service ${ }^{11}$ e pela Farmacopéia dos EUA ${ }^{10}$, desde 1970 , em substituiçăo ao meio líquido de Sabouraud ${ }^{2}$.
Medidas de precaução devem ser tomadas para evitar a contaminação do ambiente onde săo processados produtos da complexidade dos imunobiológicos. Especial atenção deve ser dispensada ao treinamento do pessoal técnico que trabalha nessas áreas, quanto à higiene pessoal $\mathrm{e}$ à utilizaçăo correta de vestimentas próprias para atividades em áreas controladas, por constituir-se no principal veiculador de partículas viáveis, seja esporos fúngicos ou bactérias.

\section{MATERIAL E MÉTODOS}

\section{Ambientes}

Com vistas ao isolamento e identificaçăo da microbiota fúngica, foram selecionados três ambientes: a) ambiente aberto (AA): sala utilizada

\footnotetext{
- Divisăo de Microbiologia e Imunologia do Instituto Butantan - Av. Vital Brasil, 1500 - 05504 - Sza Paulo, SP Brasil.

* Laboratório de Micologia Médica da Faculdade de Medicina da Universidade de São Paulo - Av. Dr. Arnaldo, 455 - 01246 - Såo Paulo, SP - Brasil.
} 
para várias finalidades, desde tarefas em bancadas livres até operaçőes em capelas de fluxo laminar, onde se realiza o preparo de meios de cultura líquidos; b) ambiente fechado, sem filtração de ar (AF): câmara asséptica convencional, sem antecâmara; c) ambiente fechado, com filtração de ar ambiente (AF/A): área limpa com antecâmara, dispondo de pressão positiva, filtros absolutos terminais tipo HEPA e vestiários assépticos, onde se processa o envasamento de produtos biológicos.

\section{Meios de cultura}

Foram empregados no isolamento da microbiota fúngica os meios ágar-Sabouraud e ágar-soja ${ }^{6}$ enriquecidos com $0,2 \%$ de extrato de levedura, sem cloranfenicol, distribuídos em placas de Petri de $15 \mathrm{~cm}$ de diâmetro.

\section{Exposição de placas de Petri/meios de cultura}

$O$ isolamento foi feito através de três exposições com meios de cultivo adequados, a intervalos de três meses, antes e após as manobras técnicas de cada área. $O$ tempo de exposição foi de 30 min. e a incubaçăo realizada à temperatura ambiente, por período de até $72 \mathrm{~h}$.

\section{Isolamento de colônias}

As colônias crescidas nos cultivos das placas de Petri foram semeadas em tubos contendo ágar-soja e ágar-Sabouraud inclinados, para isolamento e posterior identificaçăo.

\section{Identificação}

Para os espécimens isolados foram usados métodos específicos: a) Bolores - O método de cultivo em lâmina de Porto e col. ${ }^{7}$ foi adotado para identificar a micromorfologia de fungos filamentosos (bolores), utilizando-se ágar- Sabouraud dextrose, ágar-batata dextrose e ágar-fubá. Os dois últimos foram empregados visando estimular a esporulação dos fungos, as vezes insuficiente ou ausente em ágar-Sabouraud ${ }^{3}$; b) Leoeduras - Identificadas através da micromorfologia e do bioquimismo:

Micromorfologia - Obtida pelo exame microscópico dos cultivos em lâmina (método de Rivalier \& Seyde18,9, 1932, empregando-se ágarfubá acrescido de Tween-80 a $1 \%$.

Bioguimismo - Assimilaçăo de carboidratos, álcoois e nitrato de potássio, bem como a produção de urease, segundo McGinnis e col. ${ }^{5}$ (1982), e Fell e col..$^{3}$ (1984).

\section{RESULTADOS}

A Tabela 1 apresenta os dados referentes aos 24 gêneros e espécies de bolores e leveduras identificados nas três exposiçóes realizadas, permitindo comparar sua freqüência pelo número de colônias isoladas. Da microbiota fúngica presente, a maior freqüência foi observada com relaçăo aos gêneros da ordem Moniliales: Cladosporium sp. (23,88\%), Acremonium sp., Epicoccum sp., Fusarium sp. $(7,46 \%)$ e Penicillium sp. $(5,97 \%)$.

Dentre os fungos pertencentes à famf́lia Dematiaceae, foram identificados os seguintes: Alternaria sp., Cladosporium sp., Drechslera sp., Epicoccum sp. (E. nigrum), Exserohilum sp., Helminthosporium sp., Humicola sp., Periconiella sp., Sporidesmium sp. e Stemphylium sp. (Tabela 1).

\section{TABELA 1}

Freqüência de isolamento de bolores e leveduras nas três exposiçð̃es

a) Fungos com gênero e espécies da ordem Moniliales

b) Fungos incluidos nas ordens Agonomycetales Colônias e Sphaeropsidales

\begin{tabular}{|c|c|c|}
\hline 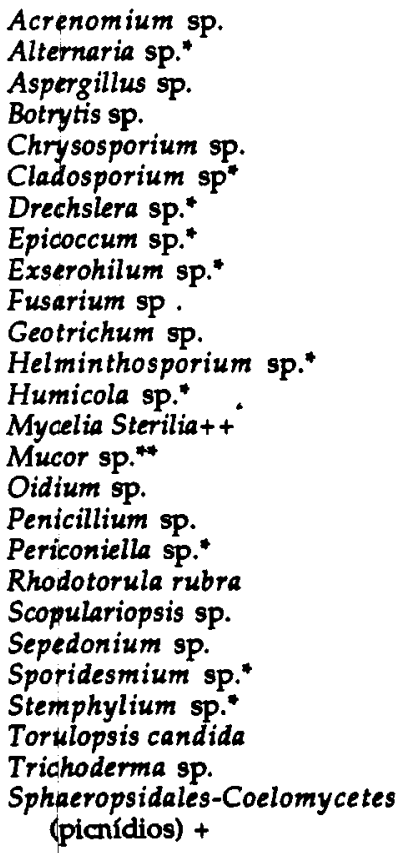 & $\begin{array}{r}5 \\
2 \\
2 \\
1 \\
2 \\
16 \\
1 \\
5 \\
1 \\
5 \\
1 \\
2 \\
1 \\
5 \\
1 \\
1 \\
4 \\
1 \\
2 \\
1 \\
1 \\
1 \\
2 \\
1 \\
2 \\
1\end{array}$ & $\begin{array}{r}7,46 \\
2,99 \\
2,99 \\
1,49 \\
2,99 \\
23,89 \\
1,49 \\
7,46 \\
1,49 \\
7,46 \\
1,49 \\
2,99 \\
1,49 \\
7,46 \\
1,49 \\
1,49 \\
5,97 \\
1,49 \\
2,99 \\
1,49 \\
1,49 \\
1,49 \\
2,99 \\
1,49 \\
2,99 \\
1,49\end{array}$ \\
\hline Total: & 67 & 100,00 \\
\hline \multicolumn{3}{|c|}{$\begin{array}{l}\text { Fungos pertencentes a famnia Dematiaceae. } \\
\text { Mucoráceo da divisão Zygomycota, famnia } \\
\text { Mucoraceae. } \\
\text { Nảo identificados até gênero. } \\
\text { Agonomycetales. }\end{array}$} \\
\hline
\end{tabular}

Fungos hialinos e pigmentados da familia Moniliaceae foram identificados até gênero: Acremonium sp., Aspergillus sp., Botrytis sp., Chrysosporium sp., Fusarium sp., Geotrichum 
TABELA 2

Distribuição do número de colônias de fungos filamentosos (bolores) e leveduras isoladas nos três ambientes, nas três exposiçōes, antes e após o trabalho

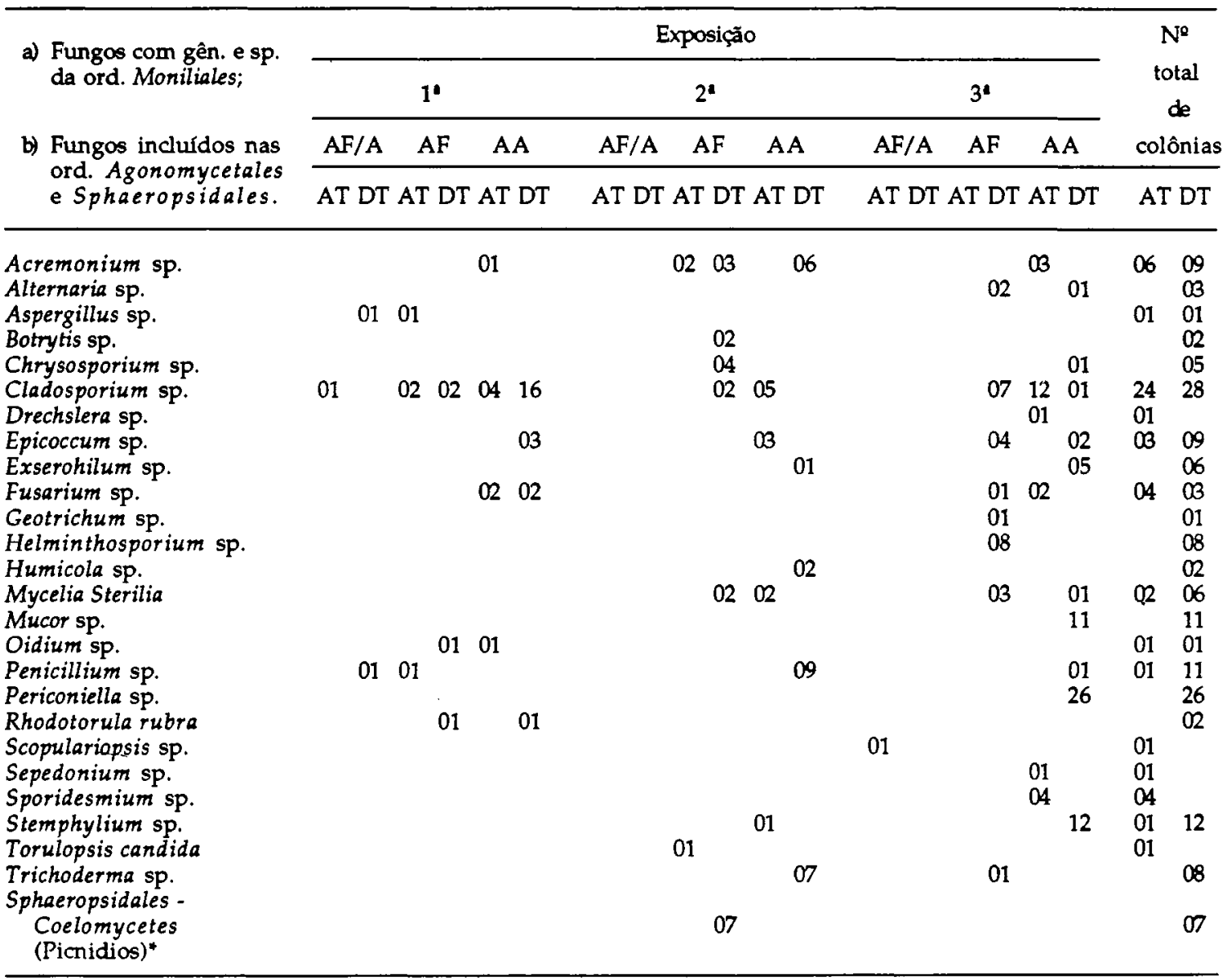

\begin{tabular}{llllllllllllllllllllll}
\hline Total & 01 & 02 & 04 & 04 & 08 & 22 & 0 & 0 & 03 & 20 & 11 & 25 & 01 & 0 & 0 & 27 & 23 & 61 & 51 & 161 \\
\hline
\end{tabular}

- = Não identificados até gênero

$\mathrm{AA}=$ Ambiente aberto

$\mathrm{AF}=$ Ambiente fechado

$\mathrm{AF} / \mathrm{A}=$ Ambiente fechado, com filtração (filtro tipo HEPA)

$\mathrm{AT}=$ Antes do trabalho

DT = Depois do trabalho

sp.,Oidium sp., Penicillium sp., Sepedonium sp., Trichoderma sp. e Scopulariopsis sp.

O único mucoráceo, Mucor sp., da ordem $\mathrm{Mu}$ corales, familia Mucoraceae, foi identificado através das características morfológicas obtidas pelo mesmo método de cultivo em lâmina, já mencionado.

As leveduras da divisão Deuteromycota, familia Crytococcaceae, identificadas até espécie foram Rhodotorula rubra e Torulopsis candida.

Estăo também incluídos nas Tabelas 1, 2 e 3:

a) Hifomicetos não identificados até gênero
$(7,46 \%)$, os quais estão incluídos na ordem Agonomycetales (Mycelia Sterilia);

b) Picnídios $(1,49 \%)$ não identificados até gênero, pertencentes à ordem Sphaeropsidales, classe Coelomycetes.

$\mathrm{Na}$ Tabela 2 foram relacionados e comparados os números de colônias isoladas nos três ambientes escolhidos, em cada exposição, antes e após a realização das manobras técnicas próprias de cada área, e pertencentes aos gêneros e espécies identificadas.

Na Tabela 3, os resultados são apresentados de modo a verificar a ocorrência dos bolores e leve- 
duras mais freqüentes nos ambientes de trabalho considerados na pesquisa, de acordo com as três exposiçסes realizadas.

\section{DISCUSSÃO}

A pesquisa de bolores (fungos filamentosos) e leveduras presentes nos três ambientes considerados assépticos levou à identificação de 67 amostras, sendo 64 bolores e três de leveduras (Tabelas 1,2 e 3). Dos bolores, 54 pertenciam a 22 gêneros da divisăo Deuteromycota, famílias Moniliaceae e Dematiaceae, identificados pelas características morfológicas apresentadas nos cultivos em lâminas.

Estes cultivos, com ágar-soja acrescido de $0,2 \%$ de extrato de levedura, a princípio ensaiados para algumas amostras de fungos filamentosos, foram posteriormente suprimidos devido à obtenção de formas irregulares de esporos ou à ausência dos mesmos.

Cinco amostras filamentosas, por não produzirem esporos, foram incluídas na ordem Agonomycetales (Mycelia Sterilia), enquanto que uma amostra produtora de esporocarpos assexuados (picnídios) foi classificada na divisão Deuteromycota, ordem Sphaeropsidales, classe Coelomycetes. Da divisão Zygomycota, ordem Muco- rales, famílias Mucoraceae, um único mucoráceo foi identificado até gênero.

As três amostras de leveduras pertenciam também à divisão Deuteromycota (Fungi Imperfecti), familia Cryptococcaceae, e foram identificadas até espécie, sendo duas Rhodotorula rubra ${ }^{3} \mathrm{e}$ uma Torulopsis candida.

Segundo as régras de nomenclatura botânica, o gênero Torulopsis é perfeitamente válido, sendo conhecido há 58 anos como levedura imperfeita (Fungi Imperfecti), não formando hifas nem pseudohifas (McGinnis", 1986). Alguns autores incluem as espécies do gênero Torulopsis no gênero Candida ${ }^{4}$. McGinnis e col. ${ }^{5}$ (1982) e McGinnis ${ }^{4}$ (1986) mantêm as espécies do gênero Torulopsis à parte das do gênero Candida, com argumentação das mais válidas e pertinentes.

$\mathrm{Na}$ terceira exposição, realizada quando as temperaturas médias eram mais elevadas, foi observada a ocorrência de maior número de isolamentos, indicando uma maior veiculação de esporos.

Com relação às exposições antes e após as manobras técnicas específicas de cada um dos ambientes, ficou comprovado um aumento no número de colônias isoladas após as manobras,

TABELA 3

Gêneros e espécies isolados nas três exposições

\begin{tabular}{|c|c|c|}
\hline \multicolumn{3}{|c|}{ Exposição } \\
\hline $1^{a}$ & $2^{a}$ & 3a \\
\hline $\begin{array}{l}\text { Acremonium sp. } \\
\text { Cladosporium sp. } \\
\text { Epicoccum sp. } \\
\text { Penicillium sp. }\end{array}$ & $\begin{array}{l}\text { Acremonium sp } \\
\text { Cladosporium sp. } \\
\text { Epicoccum sp. } \\
\text { Penicillium sp. } \\
\text { Chrysosporium sp. } \\
\text { Exserohilum sp. } \\
\text { Mycelia Sterilia } \\
\text { Stemphylium sp. } \\
\text { Trichoderma sp. } \\
\text { Humicola sp. } \\
\text { Torulopsis candida } \\
\text { Picnidios (Sphaeropsidales - } \\
\text { Coelomycetes) } \\
\text { Botrytis sp. }\end{array}$ & 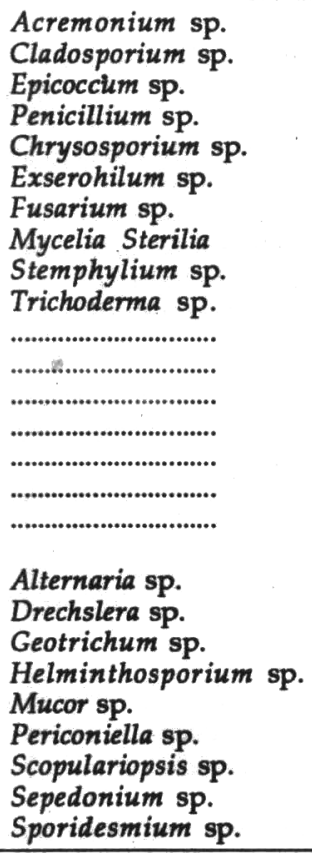 \\
\hline 8 & 13 & 19 \\
\hline
\end{tabular}


inferindo-se que a movimentação do pessoal técnico de cada área foi o seu determinante. No ambiente fechado, dotado de filtração de ar (filtro tipo HEPA), o número reduzido de colônias isoladas demonstrou claramente a segurança que a filtraçăo do ar ambiente proporciona às manobras assépticas. Nos ambientes fechados, sem filtração de ar e no aberto, ficou caracterizada a veiculação expressiva de esporos fúngicos.

Atualmente, laboratórios de produção săo pro- jetados levando em consideração a importância da existência de sistemas de filtração de ar com filtros absolutos tipo HEPA. $O$ indiscutivel aumento de custo que isto acarreta ao empreendimento justifica-se plenamente pela segurança que a filtração oferece às atividades de produção e controle de qualidade, nas quais se trabalha com matéria prima de elevado custo, difícil e demorada obtenção e de grande significado para a Saúde Pública, como é o caso dos imunobiológicos.

ALMEIDA, M.E.S. de et al. [Identification of the fungic microbiota isolated in areas considered aseptic].

Rev. Saúde públ., S.Paulo, 22: $201-6,1988$.

ABSTRACT: The isolation and identification of the fungic microbiota present in areas considered aseptic (open and/or restricted, with or without air filtration (HEPA filters) where production of immunobiologicals is carried out)were investigated. Three exposures were made with appropriate culture media, in different seasons of the year, before and immediately after the performance of the technical work. Agar-Sabouraud and agar-soy media supplemented with $0.2 \%$ yeast extract and without chloranphenicol had their efficacy tests in isolating the filamentous fungiae (molds) and yeasts in the light of the number of cultures grown and the frequency exhibited by the various specimens isolated. Sixty-seven specimens (64 of filamentous fungiae (molds) and three yeasts) have been identified. Of the filamentous fungiae (molds), 54 belonged to 22 genera of the division Deuteromycota, families Moniliaceae and Dematiaceae, five were included in the order Agonomycetales (Mycelia Sterilia), and one was classified in the order Sphaeropsidales, class Coelomycetes. Of the division $Z y$ gomycota, order Mucorales, family Mucoraceae, only one specimen could be identified as to genus. The three yeasts, belonging also to the division Deuteromycota (Fungi Imperfecti), family Cryptococcaceae, were identified as two Rhodotorula rubra and one Torulopsis candida. It was demonstrated that the number of colonies isolated in the areas studied increased after the performance of technical maneuvers and that air filtration by 'means of HEPA filters, reducing to number of colonies isolated in restricted areas, increased safety and is consequently recommended for working areas where successful or unsuccessful results depend on a low incidence of contamination.

UNITERMS: Fungs, isolation. Yeasts, isolation. Environment, controlled. Asepsis. Environmental microbiology.

\section{REFERENNCIAS BIBLIOGRÁFICAS}

1. BERLESE, A. N. I funghi diversi dai sacaromiceti e capaci di determinare la fermentazione alcoolica. G.Vitic.Enol., 3: 52-5, 1984

2. COX, C. B.; FEELEY; J. C.; PITTMAN, M. Sterility testing: detection of fungi and yeast in the presence of preservatives. J. biol. Stand., 1: 11-9, 1973.

3. FELL, J. W.; STATZELL, A.; AHEARN, D. G. Genus 10. Rhodotorula Harrison. In: Kreger-van Rij, N. J. W. The yeasts: a laxonomic stucty. $3^{\text {tr }}$ ed. Amsterdam, Elsevier Science Publ., 1984, p. 893-905.

4. McGINNIS, M. R. Some yeasts of medical importance. In: International Conference on Mycoses, $4^{\text {th }}$, Washington, D. C., 1986. Proceedings. Washington, D. C., Pan American Health Organization, 1986. p. 136- 46. (PAHO - Scientific Publication, 479).

5. McGINNIS, M. R.; D'AMATO, R. F.; LAND, G. A. Identification of yeasts. In: McGinnis, M. R.; D'Amato, R. F.; Land, G. A. Pictorial handbook of medically importans fungi and aerobic actinomycetes. New York, Praeger Publ., 1982. p. 126-37.
6. PITTMAN, M. \& FEELEY, J. C. Sterility testing: detection of fungi and yeast contamination in biological preparations, 1962. In: Intemational Congress for Microbiological Standartizadtions, $7^{\text {th }}$, London, 1963. Proceedings. London, 1963. p. 207-14.

7. PORTO, E.; TAKAHASHI, N.; HEINS, E. M.; LACAZ, C. da S. Nuevo método para microcultivo de hongos. Rev. argent. Micol., 4: 24-9, 1981.

8. RIVALIER, E. \& SEYDEL, S. Culures minces sur lames gelosés colorés \& examinées "in siru" en preparations definitives pour l'étude des Cryptogames microscopiques. C. R. Soc. Biol., 40: 181-4, 1932.

9. RTVALIER, E. \& SEYDEL, S. Nouvesu procedé de culture sur lames gélosés appliqué a l'éude microscopique des champignons des teignes. Ann. Parasiol. hum. comp., 10: 444-52, 1932.

10. STERIITY test. In: Phamacopeia of the United States of America. 20th ed. Rockville, Md, US Pharmacopeial Convention, Inc., 1980. p. 878-81. 
11. US PUBLIC HEALTH SER VICE REGULATIONS. Code of Federal Regulations, Title 21, Parts 600-799. Washington, D.C., Office of the Federal Register, 1980. p. 39-43.

12. WORLD HEALTH ORGANIZATION. Working Group on General Requirements for the Sterility of Biological Substances, Geneva, 1959. Requirements for biological substances, 6. General requirements for the sterility of biological substances; report. Geneva, 1960. (Technical Report series, 200).

Recebido para publicaçdo em $5 / 11 / 1987$ Reapresentado em 28/3/1988 Aprovado para publicaçdo em 4/4/1988 